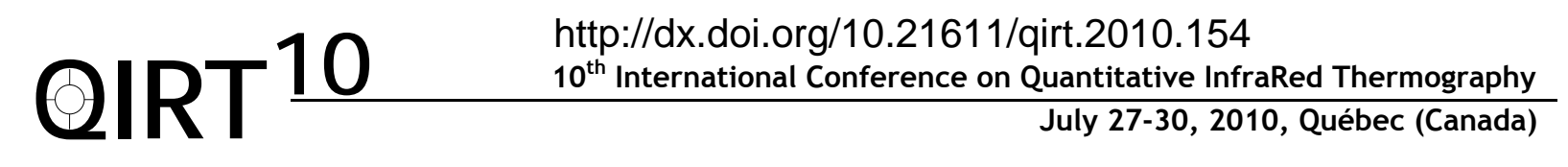

\title{
Defect detection of pipes using shearography and lock-in infrared thermography
}

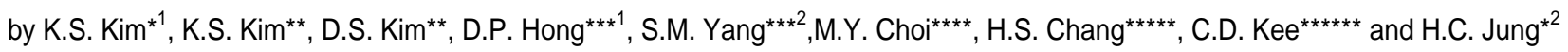 \\ *1Dept.of Mechnical Design Eng., Chosun Univ., 375 Seosuk-dong, Dong-gu, Gwangju, Korea, \\ gsckim@chosun.ac.kr \\ **Dept. of Advanced parts and Materials Eng., Graduate School, Chosun Univ., 375 Seosuk-dong, Dong-gu, \\ Gwangju, Korea, kyungsukorea@naver.com \\ $\star \star \star^{1}$ Division of Precision Mechnical Eng., Chonbuk National Univ., 664-14 1Ga Duckjin-dong, Duckjun-gu, Jeonju, \\ Jeonbuk, 561-756, Korea, hongdp@chonbuk.ac.kr \\ $\star * \star^{2}$ Division of Precision Mechnical Eng., Chonbuk National Univ., 664-14 1Ga Duckjin-dong, Duckjun-gu, Jeonju, \\ Jeonbuk, 561-756, Korea, yangsm@chonbuk.ac.kr \\ ***Korea Research Institute of Standard and Science (KRISS), P. O. BOX 102 Yuseong, Daejeon, 305-600, Korea, \\ mychoi@kriss.re.kr

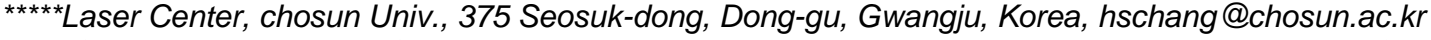 \\ $\star \star \star \star \star \star$ School of Mechanical System Eng., chonnam National Univ., 300 Yongbong-dong, Buk-gu, Gwangju, Korea, \\ cdkee@chonnam.ac.kr \\ *2Dept.of Mechnical Design Eng., Chosun Univ., 375 Seosuk-dong, Dong-gu, Gwangju, Korea, yonggary@ieee.org
}

\section{Abstract}

In this study, shearography and infrared thermography were used for measuring the wall thinning defects of the nuclear power plants pipes. It is very important to measure the wall thinning defects inside the pipes, because the wall thinning defects cause damage or destruction of the pipes. The defects are mainly occurred by the effect of the flow accelerated corrosion of fluid which flows in pipes. As a result of experiment, length and depth of the defects which are located in the pipes was accurately measured. So, the wall thinning defects are evaluated quantitatively by using shearography and infrared thermography.

\section{Introduction}

Shearography can measure the slope of the deformation by appropriately organizing the optical interferometer and is very excellent to measure a defect of objects [1]. Infrared thermography is a non-contact and non-destructive technique which can measure temperature variations of object surface. Defects detection of infrared thermography by Lock-in method is possible for measuring a temperature evolution of object by thermo elastic effects. Thus, these techniques have been developed as a non-contact and non-destructive evaluation method which can be applied to NPP pipes [2, 3]. In this paper, the wall thinning defects of NPP pipes are evaluated quantitatively by using shearography and infrared thermography.

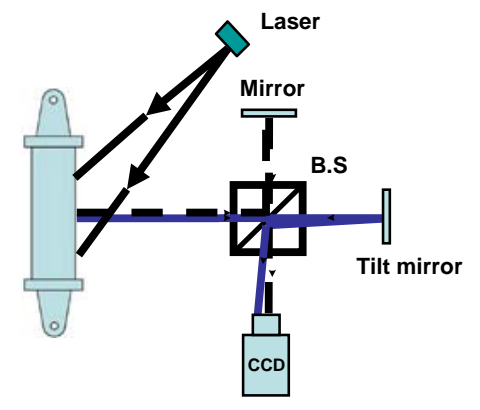

Fig. 1. Shearography system

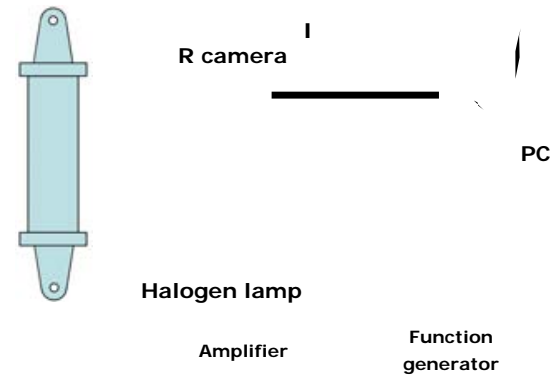

Fig. 2. Lock-in photo-infrared thermography

\section{Experiment and results}

The working principle of the experimental set-up for Shearography is shown in the Fig. 1. It consists of Nd:YAG laser system of wavelength $\lambda=532 \mathrm{~nm}$, Shearography sensor, excitation device and image processing program. The specimen was illuminated by the laser beam and the internal pressure was applied to the specimen for deformation 
measurement. The inner defects of straight pipes were measured by changing the internal pressure by shearography (shearing amount $=5 \mathrm{~mm}$ in y axis direction). The gap of pressure is $0.02 \mathrm{MPa}$ from $0.02 \mathrm{MPa}$ to $0.1 \mathrm{MPa}$ to measure the defects of the straight pipe specimen with the wall thinning defects. The experimental results of all specimens were acquired using the profiles of $y$-axis direction. The phase map and deformation of a straight pipe at different pressures are shown in the Fig. 3 and Fig. 4, respectively. The obtained results are also compared to the reference data which are obtained from the deformation measurement of the specimen without wall thinning defects. After the observation of the deformation line profile, it is possible that the wall thinning defect can be revealed by the method of shearography.

Experimental system consists of the infrared camera, function generator, and a heat source (1 kW halogen lamp), as shown in the fig.2. The heat source is driven by a power controller; the source and camera are synchronized using the sinusoidal function of the function generator. During practical inspection, the blind frequency should be avoided and the optimum frequencies should be selected. There exists a frequency for each specific defect of a certain depth such that no phase difference or a very small phase difference is produced by the defect. Thus, the defect cannot be detected at this frequency, which can conveniently be called the blind frequency. There is another special frequency, called the optimum frequency, at which a certain defect produces a maximum phase difference between the healthy part and the defective part, and this frequency was selected for detecting the defects. The images of infrared thermography are shown in Fig. 5. The defect of the specimen was not detected at over $450 \mathrm{mHz}$. From this result, the optimum frequency according to the defect depth was found and compared with the real value. The error rate between the measured value and the real defect value of the specimen is about $7 \%$.

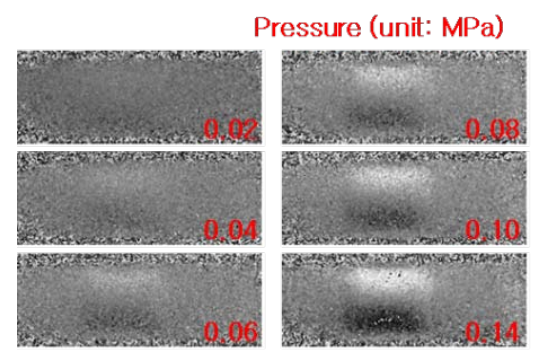

Fig. 3. Phase map of deformation at each pressure

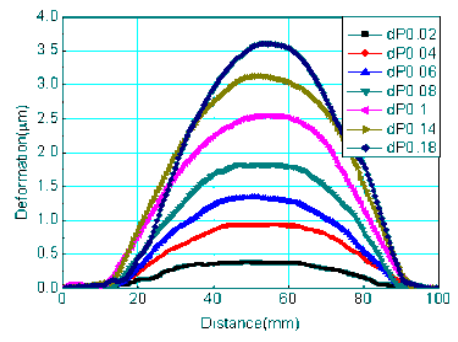

Fig. 4. Deformations of a straight pipe at each pressure

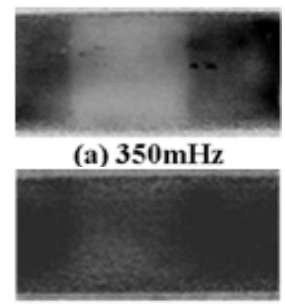

(b) $450 \mathrm{mHz}$

Fig. 5. Infrared images at each frequency

\section{Conclusion}

In conclusion, the inspection techniques were proposed using shearography and infrared thermography to compensate the technical and economical losses of the existing non-destructive technique. From the experiment results, it was accurately measured the length and depth of wall thinning defect which are located inside the pipes. The shearographic result shows that the value of deformation increases as the wall thinning thickness decreases. We determined the sizes and locations of the wall thinning defects using the shearing-phase technique, where the slope of the phase contrast at the optimum thermal wave frequency is extracted by subtraction between adjacent pixels. The wall thinning defect was parallel to pipe axis with $118 \mathrm{~mm}$ in length from the inner surface of the pipeline. The depth of defects of the specimen was determined $3.45 \mathrm{~mm}$ by thermal diffusion equation. The location and width detection were less than $5 \%$. We think the detection resolution may be improved with a high resolution camera or by capturing a close-up image of the inspection area. Although defect sizing is currently achieved using a high-performance image processing technique and this method will make it possible to easily calculate the sizes and locations of defects with high accuracy.

\section{ACKNOWLEDGEMENT}

This work was supported by Nuclear Research \& Development program of National Research foundation of Korea (NRF) funded by Ministry of Education, Science \& Technology (MEST) (Grant code: 2009-0082786)

\section{REFERENCES}

[1] Steinchen, W. and Yang, L., "Digital Shearography: Theory and Application of Digital Speckle Pattern Shearing interferometry," Washington, SPIE Press, p. 149 154, 2003.

[2] Choi, M. Y., "The utilization of non-destructive testing and defects diagnosis using infrared thermography," Journal of the Korean Society for Nondestructive Testing, vol. 24(5), p. 525 531, 2004. 